\title{
BMJ Open Publicly available apps for cancer survivors: a scoping review
}

\author{
Rosalind Adam (D) , ${ }^{1}$ Drew McMichael, ${ }^{1}$ Daniel Powell, ${ }^{2}$ Peter Murchie (D) ${ }^{1}$
}

To cite: Adam R, McMichael D, Powell D, et al. Publicly available apps for cancer survivors: a scoping review. BMJ Open 2019;9:e032510. doi:10.1136/ bmjopen-2019-032510

- Prepublication history and additional material for this paper are available online. To view these files, please visit the journal online (http://dx.doi. org/10.1136/bmjopen-2019032510).

Received 21 June 2019 Revised 04 September 2019 Accepted 10 September 2019

Check for updates

(c) Author(s) (or their employer(s)) 2019. Re-use permitted under CC BY-NC. No commercial re-use. See rights and permissions. Published by BMJ.

${ }^{1}$ Academic Primary Care, Institute of Applied Health Sciences, University of Aberdeen, Aberdeen, UK ${ }^{2}$ Aberdeen Health Psychology Group, Institue of Applied Health Sciences, Rowett Institute, University of Aberdeen, Aberdeen, UK

Correspondence to

Dr Rosalind Adam;

rosalindadam@abdn.ac.uk

\begin{abstract}
Objectives To review the nature and scope of apps targeting individuals living with and beyond cancer. Design Scoping review, searching the two largest app stores, Google Play and Apple's App store. App descriptions were exported verbatim, and summarised descriptively, thematically and by content coding.

Results We included 151 apps targeting individuals living with and beyond cancer. Most targeted all cancer types $(n=89,58.9 \%)$ or breast cancer $(n=22,14.6 \%)$ and originated in the USA ( $n=68,45.0 \%)$. The country of origin was unclear for 31 (20.5\%) apps. Most apps were developed by commercial companies/private individuals $(n=64,43 \%)$ or non-profit organisations $(n=30,19.9 \%)$ and marketed apps in terms of fighting metaphors, navigating a journey and becoming empowered to take control.
\end{abstract}

App content could be summarised under five main categories: (1) imparting information about cancer; (2) planning and organising cancer care; (3) interacting with others (including others affected by cancer and healthcare professionals); (4) enacting management strategies and adjusting to life with or beyond cancer and (5) getting feedback about cancer management, for example, by sharing self-monitoring reports with professionals. We found some apps describing 'cures' for cancer or selling products, such as alkaline waters to cancer survivors. Conclusions Apps are currently available via online stores that cover a large spectrum of cancer survivorship activities. The effects of such apps on clinical consultations, patient work/burden and clinical outcomes merit further attention. Most apps are developed by commercial organisations, and promises of empowerment in the 'fight' against cancer are tempered by the potential for exaggerated claims and exploitation.

\section{INTRODUCTION}

The number of individuals living with and beyond cancer (also known as cancer survivors) is increasing. ${ }^{12}$ In the UK, it is estimated that the number of cancer survivors will grow by approximately one million every decade, from 2.1 million in 2010 to 5.3 million in $2040 .{ }^{2}$ Cancer is increasingly being regarded as a chronic disease due to the growing number of individuals who are living with cancer or surviving cancer $^{3}$ with long-term symptoms $^{4}$ and late effects of cancer treatment. ${ }^{5}$ Cancer survivors can experience increased physical, psychological and social issues after
Strengths and limitations of this study

- Scoping review categorising and summarising a wide range of apps available for cancer survivors on on-line stores.

- Content and thematic analysis based on verbatim descriptions from the stores.

- Individual apps not downloaded or quality assessed.

their diagnosis, ${ }^{6}$ accompanied by a range of unmet needs. ${ }^{7}$ There is growing political and clinical interest in using digital technologies to deliver efficient, high-quality care for cancer survivors ${ }^{8}$ and to empower patients to perform self-management activities. ${ }^{9}$

The market for apps, including health apps, is growing rapidly, ${ }^{1011}$ with an estimated 318 000 health apps available in $2017 .{ }^{12}$ It is estimated that over 200 health apps are added daily to app stores. ${ }^{13}$ Against this changing technological environment, attempts to summarise and evaluate healthcare apps in traditional systematic reviews are limited by the relatively small proportion of technologies, which are reported in published literature. ${ }^{1415}$ An alternative strategy has been to identify and review apps that are available for download via on-line stores. ${ }^{16-18}$

In 2012, Bender et al searched on-line stores to characterise the purpose and content of apps focusing on any aspect of cancer. ${ }^{19}$ Of 295 cancer apps, most were limited in their scope, focusing primarily on providing information and raising awareness about cancer in general and promoting/fundraising for charities. ${ }^{19}$ In 2014, Kassianos et al searched on-line stores for melanoma detection apps, identifying 39 apps. Most gave education or advice about melanoma, ultraviolet radiation exposure and skin self-examination strategies. ${ }^{20}$

Relatively, little is known about apps targeting people living with and beyond cancer. Dahlke et $a l^{21}$ conducted a review to identify behaviour change techniques $(\mathrm{BCTS})^{22}$ embedded in cancer survivorship apps, searching app stores in November 
$2013 .^{21}$ Eighteen BCTs were present across 65 apps, including providing instruction, tailoring (e.g. adjusting the information delivered based on user input), personalisation (e.g. the user can select elements specific to them such as disease type) and prompting intention formation. What was less clear were the range of behaviours targeted, the aims and scope of the apps, how the BCTs were operationalised and organised, and where the technology itself might add value to survivorship care.

The aim of this review is to characterise apps targeting individuals living with and beyond cancer that are currently available for download via the two major app stores (Google Play and Apple's App Store), which have been estimated to contain over $90 \%$ of all apps. ${ }^{20}$ The review will provide a summary of the apps' advertised components, stated aims and technological features. We aim to categorise and organise the apps such that clinicians, app developers and policy-makers can make sense of the current international app market for people living with and beyond cancer.

\section{METHODS}

We performed a scoping review, ${ }^{23}$ searching Apple's App Store and Google Play to identify apps targeting people living with and beyond cancer, and used content anal$\mathrm{ysis}^{24}$ to characterise advertised content. Scoping reviews differ from traditional systematic reviews in that they map a topic in order to communicate the breadth and depth of the field ${ }^{25}$ and do not tend to involve formal quality assessment of the evidence. ${ }^{23}$ They describe the 'extent, range and nature' ${ }^{23}$ of the available evidence, and set it in context in terms of current understanding. Scoping reviews require analytical interpretation of the subject area. ${ }^{26}$ They are particularly useful when synthesis involves non-research material, ${ }^{26}$ and for emerging areas of research.

In this scoping review, we did not download and interact with the apps or test quality or functionality. In a previous review, Kassianos et al used app store summaries and were able to yield detailed descriptions of melanoma app content. ${ }^{20}$ Based on older reviews, ${ }^{1921}$ we expected to find a large number of apps, and in this rapidly changing field, the time required to interact with each app would lead to significant delays in communicating our findings. We wished to include paid apps, and had limited resources to buy individual apps for multiple authors. We also wished to include any apps affiliated with specific centres or clinical trials which would require log in credentials.

\section{Search strategy}

Initial searches were conducted by two authors, DM and RA, who refined the search criteria. The lead author then searched the two leading app stores, Apple's App store (we used an iPhone with iOS operating system), and Android's Google Play (we used a Personal Computer (PC) with Windows operating system) in September 2018 using the keywords 'cancer', 'cancer survivor' and 'cancer survivorship'.

\section{Inclusion and exclusion criteria}

We included: apps aimed at patients living with and beyond cancer; free and paid apps from any country; apps that included prediagnosis support and information (so long as they also specifically targeted individuals living with and beyond cancer); and apps covering more than one clinical condition, so long as cancer was a named condition.

We excluded: apps unavailable in English or without English descriptions; simple awareness raising or prediagnosis apps (apps raising awareness of symptoms that were potentially indicative of cancer or risk assessment tools, skin/mole checking apps for individuals without a diagnosis of skin cancer, simple factsheets about a certain cancer type or glossaries); and recipe and diet apps that were not specifically targeting patients living with and beyond cancer.

Apple's App store operates a 'continuous scroll' function, meaning that the store loads content continuously to return results. General search terms or keywords (eg, 'cancer') can result in 'endless scrolling' or 'infinite scrolling', where results are continuously returned without an apparent endpoint. Our initial scoping searches showed that apps became much less relevant after the first few hundred results, and we decided to limit our search of Apple's App store to the first 500 results for the term 'cancer'. The on-line stores are not set up to allow search results to be exported, and decisions about inclusion and exclusion were made by a single author (RA). Eligibility was determined from the descriptions of the apps within the app stores. Descriptions of the final apps selected for inclusion were reviewed by a second author (DM) to ensure that apps met the eligibility criteria.

\section{Data extraction and synthesis}

A data extraction form was created in Microsoft Excel and two researchers (RA and DM) independently extracted data from all apps that met the inclusion criteria. Data were obtained from the stores' on-line app descriptions, principally the narrative text, but notes were also taken based on screenshots of the apps within the store. These notes were added to summarise any visible content from the screenshots which was in image form but not directly mentioned in the app description. Verbatim text from the screenshots was imported where available. We searched for and visited developer websites when available in order to gather background information on the app, particularly with respect to the nature of the organisation involved in app development (eg, non-profit organisation or charity, commercial, academic) and country of origin.

Data were extracted on: cancer type(s); name of owner and/or developer (sometimes the same); country of origin; operating system (Apple/Android/both); fee to download; type of owner (charity, commercial, academic institution, healthcare provider and combination); 
number of downloads (available on Google Play only); star rating and the presence/absence of a statement about clinical or scientific input into app development. Data on number of downloads, and star ratings were extracted by the lead author alone, as this was judged to be a changing parameter. Data were imported into SPSS V.24, and descriptive statistics were calculated.

The text description of the app given in the on-line store was copied verbatim and imported into Microsoft Word. We used content analysis ${ }^{24}$ and thematic analysis ${ }^{27}$ to organise, categorise and synthesise qualitative data. App descriptions and accompanying notes were imported into NVivo V.11. Data familiarisation took place by reading and rereading app descriptions. Initial codes were generated by the lead author in order to organise the data into meaningful groups and these were discussed with a second author (DP). ${ }^{28}$ Codes were sorted into categories, based on how the codes were related and linked. Each app was then analysed independently by two reviewers (RA and DM) to categorise the advertised content. The reviewers were alert to any content which did not fit the categories. We also analysed themes within the language used to describe the apps. Reviewers met after the data categorisation exercise and compared results, reaching consensus by discussion.

\section{Patient and public involvement}

We did not involve patients or the public in this scoping review, but rather plan to use the results to inform technology codesign projects which involve patients and the public.

\section{RESULTS}

We screened 1265 apps and included 151 in our final synthesis (see flow chart, figure 1). The main reasons for exclusion were apps not specifically targeting cancer survivors, only targeting clinicians or not relating to cancer. Four apps (Cancer Stage IV Cure Methodology, Driver, Inspire and NIH Breast Cancer Information) became unavailable during our data analysis process between 8 September 2018 and 24 May 2019, and were excluded because we were unable to return to the on-line descriptions to check accuracy during dual data extraction.

A full list of included apps, data on star ratings and the raw data used in our analyses are included in the online supplementary file 1. App names will be reported in Italics throughout our results.

\section{App demographics}

Characteristics of the apps are provided in table 1. Of note, most of the apps covered all cancer types $(n=89$, $58.9 \%)$ or were specific to breast cancer $(\mathrm{n}=22,14.6 \%)$. Over $90 \%$ were free to download. Apps were developed by a mix of private companies/individuals $(\mathrm{n}=64,43 \%)$, charity/non-profit organisations $(n=30,19.9 \%)$, healthcare organisations $(\mathrm{n}=15,9.9 \%)$ and academic institutions $(\mathrm{n}=8,5.3 \%)$. We found apps sharing the same developer and with similar content, but differing by cancer type: Eight apps were developed by '@point of care', and five apps developed by 'Self-care catalysts', both commercial developers based in the USA. The nature of the developer could not be determined for 16 apps (10.6\%).

Unlike Apple's App store, Google Play provides statistics for the number of app downloads. Of the 104 apps available on Google Play, 45 apps $(43.3 \%)$ had been downloaded less than 100 times. Five apps (Cancer.net mobile, MD Anderson mobile, Cancer fighting food, Cancer Curing foods and My Cancer Coach) had between 10000 and 50000 downloads. One app, Belong Life, had over 50000 downloads. Belong Life markets itself as an 'information sharing platform', featuring an on-line social network of individuals with cancer, healthcare professionals who answer questions, access to personalised information and a clinical trial matching service.

\section{Themes within on-line descriptions of the apps}

Verbatim app descriptions and text from screenshots ran to over 30000 words. Three prominent themes were: Fighting for Life, Navigating a Journey and Being Empowered to Take Control. Examples of app descriptions fitting these themes, with quotations, are included in table 2. Fighting metaphors were observed within a range of apps and were sometimes contained within the app title (eg, Attack Cancer using Hypnosis E Guided Imagery/Meditation, Cancer Defeated and Cancer Fighting Foods). In fighting metaphors, cancer was depicted as an enemy invader and surviving cancer as a battle. Metaphors about fighting and battles were prominent in apps promoting healthy eating or specific 'cancer-fighting' foods or diets.

Cancer was often compared with a journey and apps marketed themselves as tools to help navigate that journey. Apps that incorporated social networking often emphasised that the cancer journey did not have to be navigated alone. Social networking was suggested as a source of knowledge and emotional support. Many apps promoted peer comparison, and emphasised that others around the world were facing very similar problems, with some apps referring to 'others like you'. There was a sense within language used that cancer was associated with loss of control, and that downloading and interacting with an app was a method of empowerment and taking back control. Apps were marketed to individuals as a method of becoming actively involved in self-management.

\section{Content analysis}

The apps offered content that could be summarised under five main categories: (1) imparting information about cancer; (2) planning and organising cancer care; (3) interacting with others (including others affected by cancer and healthcare professionals); (4) enacting management strategies and adjusting to life with or beyond cancer and (5) getting feedback about cancer management. The specific app features that support each of these activities are summarised in table 3 and discussed below. 


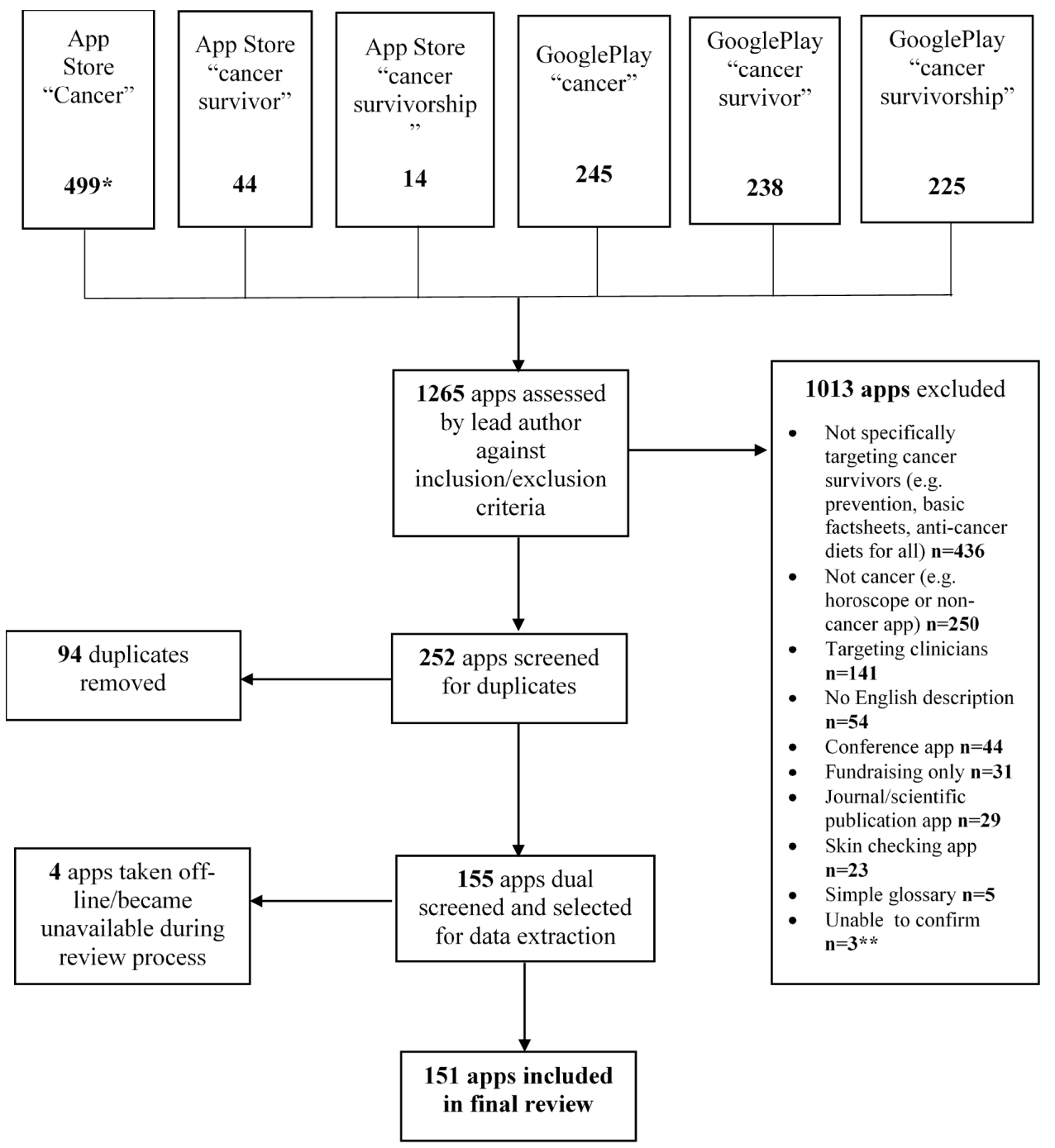

*Apple's app store was searched up to and not including the $500^{\text {th }}$ app on the on-line store: after several hundred apps, the apps became less relevant to our review

** There were three apps which we were unable to find again after the initial searches: two (faith.org; and ovarian cancer treatments (things to do) seemed to be removed from the store, and there was an administrative error during recording the name of the third app, which led to a missing field in our exclusion file

Figure 1 Identification and screening process for apps included in this review.

\section{Imparting information about cancer}

Over half the apps $(n=81,53.6 \%)$ stated in their description that they provided information or educational materials about cancer; for example, the nature of cancer, aspects of terminology related to cancer and cancer treatments. The apps presented this in various ways, including fact-sheets/written information, news feeds and updates, questions and answers, and videos. Some apps (eg, Breast Cancer Ally) provided personalised information based on user-reported characteristics, including treatments received or disease subtype. One app, Personalized Sarcoma Care, offered prognostic information to users with highgrade soft-tissue sarcoma of the limb who were going to be treated with surgery and radiotherapy. The app offered a disclaimer that it was not a medical device, not meant to be used to inform clinical decisions, and not tested for clinical usefulness. Users were instructed to discuss prognostic results with their physician.

\section{Planning and organising cancer care}

Twenty-five apps (16.6\%) allowed users to enter and store records relating to their cancer care, such as results or diaries of treatments they had received. Apps also allowed users to keep a calendar of appointments $(n=12,8.0 \%)$ and to keep lists and scheduling of medications $(\mathrm{n}=20$, 13.3\%). Three apps (MD Andersen Mobile, MyMSK and $N E D)$ allowed registered users linked to the specific cancer centre to $\log$ in and view some of their own results. Four apps (MD Andersen Mobile, MyMSK, CanHOPE cancer 
Table 1 Description of apps targeting individuals living with and beyond cancer available on Apple's App Store and Google Play

\begin{tabular}{|c|c|c|c|}
\hline Cancer types covered & $\mathrm{n}=151 \mathrm{n}(\%)$ & $\mathbf{N}(\%)$ & $\mathbf{N}(\%)$ \\
\hline All cancers & 89 (58.9) & $23(48.9)$ & $24(63.2)$ \\
\hline Breast & $22(14.6)$ & $9(19.1)$ & $5(13.2)$ \\
\hline Prostate & $9(6.0)$ & $5(10.6)$ & $2(5.3)$ \\
\hline Lung or mesothelioma & $5(3.3)$ & $2(4.3)$ & $2(5.2)$ \\
\hline Bladder or renal & $5(3.3)$ & $3(6.4)$ & $1(2.6)$ \\
\hline Liver and/or pancreas & $3(2.0)$ & $3(6.4)$ & $0(0.0)$ \\
\hline Haematological & $3(2.0)$ & $0(0.0)$ & $1(2.6)$ \\
\hline Retinoblastoma, eye or childhood cancers & $3(2.0)$ & $0(0.0)$ & $1(2.6)$ \\
\hline Colorectal & $2(1.3)$ & $0(0.0)$ & $0(0.0)$ \\
\hline Melanoma & $2(1.3)$ & $1(2.1)$ & $0(0.0)$ \\
\hline $\begin{array}{l}\text { Multiple cancers (breast and ovarian, breast, prostate and } \\
\text { colorectal) }\end{array}$ & $2(1.3)$ & $0(0.0)$ & $0(0.0)$ \\
\hline Head and neck or oral & $2(1.3)$ & $1(2.1)$ & $1(2.6)$ \\
\hline Others (testicular, ovarian, soft-tissue sarcoma, carcinoid) & $4(2.6)$ & $0(0.0)$ & $1(2.6)$ \\
\hline \multicolumn{4}{|l|}{ Type of developer } \\
\hline Commercial or private organisation & $65(43.0)$ & $23(48.9)$ & $20(52.6)$ \\
\hline Charity or non-profit organisation & $30(19.9)$ & $12(25.5)$ & $1(2.6)$ \\
\hline Unclear & $16(10.6)$ & $2(4.3)$ & $11(28.9)$ \\
\hline Healthcare organisation & $15(9.9)$ & $7(14.9)$ & $3(7.9)$ \\
\hline Academic organisation & $8(5.3)$ & $2(4.3)$ & $2(5.3)$ \\
\hline Clinical or research societies/networks or government & $4(2.7)$ & $0(0.0)$ & $0(0.0)$ \\
\hline Partnership of various types of organisation & $13(8.6)$ & $1(2.1)$ & $1(2.6)$ \\
\hline \multicolumn{4}{|l|}{ Country of origin } \\
\hline USA & $68(45.0)$ & $25(53.2)$ & $5(13.2)$ \\
\hline Unclear & $31(20.5)$ & $7(14.9)$ & $17(44.8)$ \\
\hline UK, Ireland or Gibraltar & $15(9.9)$ & $8(17.0)$ & $1(2.6)$ \\
\hline Multinational & $7(4.6)$ & $2(4.3)$ & $1(2.6)$ \\
\hline India & $7(4.6)$ & $0(0.0)$ & $5(13.2)$ \\
\hline Canada & $5(3.3)$ & $0(0.0)$ & $2(5.3)$ \\
\hline Malaysia or Singapore & $5(3.3)$ & $1(2.1)$ & $3(7.9)$ \\
\hline Australia & $4(2.6)$ & $2(4.3)$ & $0(0.0)$ \\
\hline The Netherlands & $3(2.0)$ & $1(2.1)$ & $1(2.6)$ \\
\hline $\begin{array}{l}\text { Others (France, Germany, Hungary, Morocco, Pacific } \\
\text { Islands, Spain) }\end{array}$ & $6(4.0)$ & $1(2.1)$ & $3(7.9)$ \\
\hline \multicolumn{4}{|l|}{ No of downloads (Google Play data only for 104 apps) } \\
\hline$<100$ & $45(43.3)$ & & $17(44.7)$ \\
\hline $100-500$ & $24(23.1)$ & & $10(26.3)$ \\
\hline $500-1000$ & $9(8.7)$ & & $4(10.5)$ \\
\hline $1000-5000$ & $17(16.3)$ & & $4(10.5)$ \\
\hline $5000-10000$ & $3(2.9)$ & & $1(2.6)$ \\
\hline $10000-50000$ & $5(4.9)$ & & $2(5.3)$ \\
\hline$>50000$ & $1(0.9)$ & & $0(0.0$ \\
\hline
\end{tabular}

\section{Unique to Apple Unique to}

$\mathrm{n}=47$, Google $\mathrm{n}=38$

No (N) (all apps, N (\%)

24 (63.2)

$89(58.9)$

$3(48.9)$

$5(13.2)$

5 (3.3)

$2(4.3)$

$(5.2)$

$3(2.0)$

$3(6.4)$

$1(2.6)$

$2(1.3)$

$0(0.0)$

$0(0.0)$

Melanoma

colorectal)

Head and neck or ora

$2(1.3)$

$1(2.1)$

$1(2.6)$

Others (testicular, ovarian, soft-tissue sarcoma, carcinoid)

$65(43.0)$

$5(9.9)$

$7(14.9)$

$3(7.9)$

$4(2.7)$

$0(0.0)$

Clinical or research societies/networks or government

$68(45.0)$

7 (4.6)

$0(0.0)$

$2(5.3)$

$1(2.6)$

$3(7.9)$

Continued 


\section{Cancer types covered}

Price to download (£ Sterling)

\begin{tabular}{lccc} 
Free & $140(92.7)$ & $46(97.9)$ & $34(89.5)$ \\
$<£ 9.49$ Google Play/<£12.99 App store & $9(6.0)$ & $1(2.1)$ & $2(5.3)$ \\
$>£ 12.99$ & $2(1.3)$ & 0 & $2(5.3)$ \\
\hline
\end{tabular}

support and Pratheeksha) allowed registered patients to view or change appointments.

Nine apps listed cancer clinical trials that may be relevant to individuals living with cancer, and one (Belong Life) offered a clinical trials matching service based on parameters entered by the user. Two apps supported survivorship care plans (SCPs). My Care Plan suggested that users should input data to create their own SCP, and then complete it with their oncologist. The Survivor Care app allowed registered patients with testicular cancer to use the app to read a QR code (quick response code or matrix bar code), generated by their specialist, which gave them access to a personalised care plan.

\section{Interacting with others}

Twenty five apps (16.6\%) offered access to an on-line community (social network) of other individuals with cancer, promoting these networks as sources of support and information. Four of these (Boobytrapp, Breast Cancer Health, Breast Cancer Social and Cnected) advertised a matching service in which users could be matched with other users or groups based on characteristics such as cancer type, stage, treatments and interests.

Apps also offered interaction with healthcare professionals: three apps (Belong.life, Breast Friends app and Cancer Connect) listed the ability to message or ask questions of professionals who were linked to the app platform. Four apps (Medocity's iCancer Health, MD Andersen Mobile, MyMSK and Pratheeksha) allowed users to login and send messages to their linked care team.
Enacting management strategies and adjusting to life with or beyond cancer

Specific aspects of self-management supported by apps include symptom tracking and monitoring; setting alarms or reminders to take medications regularly, tracking and adjusting diet and physical activity levels, using psychological and complementary approaches, and knowing when to seek medical attention for chemotherapy side effects.

Twenty-nine apps (19.2\%) allowed users to track their symptoms: mainly fatigue, pain, mood changes, nausea and sleep problems. Some suggested monitoring physical or physiological parameters, including pulse, blood pressure and weight, and some allowed customisation, letting the user decide which symptoms/parameters to monitor. The recommended frequency of self-monitoring varied, with some promoting weekly input, some apps suggesting on-demand tracking when symptoms were experienced, and others not specifying particular intervals for self-monitoring. Apps used a number of rating scales, including touch-screen sliders, and faces rating scales. The data were used to provide graphs and output reports (see 'feedback' below).

Fifteen apps (9.9\%) gave advice about diet and/or exercise after a cancer diagnosis, with five allowing users to track their exercise or dietary activities. Four apps offered integration with wearable fitness trackers.

Complementary and alternative therapies were a prominent component of 12 apps (7.9\%), which gave instructions on relaxation techniques, provided 'healing' music playlists, and taught guided imagery, visualisation,

Table 2 Themes present within APP marketing statements with example quotations

Theme Example app (name in Italics) with quotations from on-line app store

Fighting for life Twist out cancer: '(get to) know other cancer survivors who fought with odds and kicked cancer in the butt!' 'Whip cancer provides people with the power to instantly and accurately picture the cancer cells they want to expel from their bodies... Whip Cancer is a powerful tool to help you become relaxed and thus feel empowered while battling your cancer.'

Navigating a Breast Cancer healthline: 'You're not on this journey alone. Are you facing a diagnosis? Already in journey treatment? Remission? We'll connect you with people just like you, at the same stage in the journey'. 'The BigC-Connect platform has been designed to help survivors of cancer on their journey to survival.'

Being empowered 'Hearing that you have been diagnosed with breast cancer can turn your world upside down. The OWise to take control breast cancer app can help you regain control during the chaotic times of illness and treatments' 'Blood Cancer Storylines is filled with great tools to help you take control of your health.' 


\begin{tabular}{|c|c|c|}
\hline Survivorship activity & App feature & feature \\
\hline $\begin{array}{l}\text { Imparting information } \\
\text { about cancer }\end{array}$ & $\begin{array}{l}\text { Delivers information about the nature of cancer, cancer terminology, treatment } \\
\text { approaches and services. Apps present information as text, news feeds/ } \\
\text { updates, videos and question/answer formats. }\end{array}$ & $81(53.6)$ \\
\hline \multirow{6}{*}{$\begin{array}{l}\text { Planning and organising } \\
\text { cancer care }\end{array}$} & Upload and store personal records, for example, diaries/journals, results. & $25(16.6)$ \\
\hline & Share uploaded personal records with others. & $8(5.3)$ \\
\hline & Keep a calendar of appointments. & $12(8.0)$ \\
\hline & Login to view or change clinical appointments. & $4(2.7)$ \\
\hline & Login to remotely access clinical records or results. & $3(2.0)$ \\
\hline & Create or view survivorship care plan. & $2(1.3)$ \\
\hline \multirow[t]{4}{*}{ Interacting with others } & $\begin{array}{l}\text { Access to an on-line cancer community or social network (four offered a } \\
\text { matching service). }\end{array}$ & $25(16.6)$ \\
\hline & List of local (geographically limited) sources of peer support. & $11(7.3)$ \\
\hline & Message a linked healthcare professional. & $4(2.7)$ \\
\hline & Ask a professional within an on-line community. & $3(2.0)$ \\
\hline \multirow{4}{*}{$\begin{array}{l}\text { Enacting management } \\
\text { strategies and adjusting }\end{array}$} & Track and record-specific symptoms or physiological parameters. & $29(19.2)$ \\
\hline & Provides symptom management tips and advice. & $5(3.3)$ \\
\hline & Set alarms as reminders to take medication. & $14(9.3)$ \\
\hline & $\begin{array}{l}\text { Track fitness or diet (four apps offered integration with wearable fitness } \\
\text { trackers). }\end{array}$ & $5(3.2)$ \\
\hline $\begin{array}{l}\text { Getting feedback about } \\
\text { cancer management }\end{array}$ & Allows video or audio recording of medical consultations. & $4(2.7)$ \\
\hline
\end{tabular}

meditation, Qigong and yoga. The MeTime app, developed by University of Michigan, taught acupressure to manage fatigue in breast cancer survivors, and quoted evidence supporting its use from a randomised controlled trial. ${ }^{29}$ Three apps (Emory Awake, UNTIRE and Bubble VR) delivered programmes of psychological therapy to cancer survivors; for example, Bubble VR delivered cognitive behavioural therapy, guided imagery, meditation and mindfulness-based stress reduction within Virtual Reality. The app was linked to a focus group research study, and registered participants could use a PIN code to interact with it.

Three apps (CanAdvice+, Cancer Emergency Response Tool and For Cancer Care) specifically targeted people on chemotherapy, and sought to help users judge when to seek medical attention for side effects or problems experienced during chemotherapy. CanAdvice+ and Cancer Emergency Response Tool were linked to UK cancer centres and used the UK Oncology Nurses Society triage tool, ${ }^{30}$ whereas For Cancer Care offered generic tips and advice to manage chemotherapy side effects.

Some apps dealt with changes in body image after cancer and psychological adjustment to physical changes. Inkspiration app allowed users to 'try on' mastectomy tattoos, super-imposing tattoos onto photo uploads. The Breast Cancer Care app (BECCA) offered beauty tips alongside other information about breast cancer. 


\section{Getting feedback about cancer management}

Twenty-one apps (13.9\%) allowed users who had tracked symptoms to generate graphical summaries of their self-monitoring data for personal reflection (usually line graphs showing, for example, pain levels plotted against date/time), and to generate output reports from the data, usually by email. A central premise was that users would learn about patterns within their symptoms, and that sending their symptom reports to professionals could result in action by the professional to help with symptom management.

Apps also attempted to influence clinical encounters between users and their clinicians by allowing them to generate (sometimes from templates or lists) or store questions that they would like to ask at the next medical encounter $(\mathrm{n}=13,8.6 \%)$. Four apps (Focus on Lymphoma, My Cancer Coach, OWise Breast Cancer and Pocket Cancer Care Guide-National Coalition for Cancer Survivorship) allowed users to video or audio record their medical consultations.

\section{Clinical and/or scientific basis for App content and Apps as commercial opportunities}

Fifty-one out of 151 apps $(33.8 \%)$ cited clinical or scientific/clinical research team input into the development of the app within the on-line description. Most of these apps were developed by recognisable institutions, such as universities, clinics or charities. However, one app, Don’t Die 2 retailing at £299.99 on Google Play, had limited information about content, and stated: 'Contains new cancer approach not previously available to cancer victims. All results obtained after a rigorous 12-year study and when applied to active cancer patients show dramatic results increasing survival results.' The app was developed by a family cancer foundation (MiSong Foundation), and screenshots showed an enquiry form which users could fill in for further information. Links to the developer website were inactive.

We found a number of apps offering purchasable products to cancer survivors and apps that made claims about offering a potential cancer cure. Best Prostate Cancer Treatment opened its description by stating: 'Court Documented Proof That The Cure For Prostate Cancer \& Colon Cancer is real'. Screenshots from the app were captioned with 'PROSTATE CANCER Cure for Cancer Now Available', and the app offered treatments based on whole-leaf Aloe Vera. One screenshot showed an 'Advanced Package' with products available for US750. The app description marketed the product as follows: 'The advanced package provides specific elements to expedite the healing process. This package revitalises and engages intercellular advancement and ease in detoxification'. Cancel Cancer mentioned links between body acidity and cancer, and screenshots from the app showed order forms, products for sale, and videos about Kangen water, an alkaline water.

Three apps contained the word 'cure' in their title. Cancer Cure (retailing on Google for £28.99) offered '300 alternate healing ideas' and was recommended for 'anyone who is struggling for cancer survival'. Cancer Curing Foods (free to download) had been downloaded more than 10000 times on Google Play and offered 'top 10 fruits, vegetables and foods that can cure cancer'. Various Cancer Cures, offered free via Google Play, listed information about surgery, chemotherapy and radiotherapy as treatment options for cancer.

Cancer Wellness invited users to complete a weekly cancer survey, with links to a private clinic in the Pacific Islands (http://cancerwellnessclinic.com/our-treatmentprogram/) that offered alternative cancer treatments and supplements. In Ways to Fight Off Cancer, available on Google Play, the on-line description had statements that included 'broccoli cures cancer' and 'tomato cures cancer'... 'So What Are you Waiting For !?! Download the 'Ways Fight Off Cancer' Now!' Other potentially exaggerated claims were found in apps promoting complementary therapies and visualisation; for example, Cancer Fighting App stated (sic.) 'After working on visualisation for few weeks, the cancer tumour had shrunk to small its size and its continuous become smaller and smaller. Imagination and visualisation for creating radiant, lifelong health and happiness.'

\section{DISCUSSION}

\section{Main findings}

We reviewed 151 apps targeting individuals living with and or beyond cancer, available for download via on-line stores. The apps are often marketed in terms of fighting cancer, taking a journey and taking control. Apps are heterogeneous in terms of aims and scope, but typical content includes informational resources, diary functions, access to on-line social networks/communities and symptom-tracking capabilities linked to graphical outputs.

App owners came from a range of backgrounds (eg, non-profit organisations, academic institutions and healthcare providers), but most publicly available apps had been developed by commercial or private organisations. We were unable to discern the nature of the developer in 16 apps, despite visiting linked websites. We also found some apps that seemed to make exaggerated claims, for example, about foods that cure cancer, visualisation regimes that shrink tumours (see examples above), and apps that marketed or sold products with questionable efficacy (eg, Kangen water or Aloe Vera extract).

\section{Comparison with existing literature and implications for practice, policy and research}

Violence and journey metaphors are known to be widely used in the context of cancer and cancer fundraising campaigns, and have sparked debate. ${ }^{31}$ Both violence and journey metaphors can be perceived in both positive, and in disempowering ways. We found these metaphors to be prominent in on-line app marketing.

Apps cover some of the areas that are recommended in clinical guidelines for cancer survivorship care, ${ }^{32}$ such as 
information provision; making lifestyle changes (particularly diet and exercise), dealing with physical and psychosocial effects of cancer and its treatment, and providing SCPs. The potential usefulness of reputable apps may be undermined by the fact that they appear in stores alongside those that are potentially exploitative.

Guidelines and regulatory procedures for health apps have been introduced in the UK ${ }^{33} 34$ and USA ${ }^{35}$ but these mainly apply to apps classed as medical devices (used to diagnose, support diagnosis or clinical decision-making or make calculations to determine diagnosis or treatment), which are considered to carry the highest risks. Apps that provide education, monitor health or wellbeing, and store or transmit data without change are not subject to the same regulatory procedures. ${ }^{34}$ These types of apps can be developed quickly by anyone who wishes to ${ }^{36}$ without specific regulatory requirements.

There is increasing recognition that lack of public trust is a major barrier to the successful utilisation of data and technology to improve patient outcomes. ${ }^{37}$ In a recent review, Wyatt discussed problems with health apps, including privacy issues, poor quality content and variable accuracy, for example, in diagnosing melanoma. ${ }^{38}$ In our review, most apps were free, and it was beyond the scope of this review to determine how apps attracted revenue. Potential sources include advertising, in-app purchases and data 'harvesting'. There is increasing evidence that sharing of user data is routine in medical apps ${ }^{39}$ and that data harvesting for targeted advertising is an important source of revenue for many app developers. ${ }^{38}$

Questions remain about the clinical role of cancer apps, how they affect formal medical care and influence clinical outcomes. Some of the apps we reviewed helped users to generate lists of questions that could be taken to appointments or facilitated video or audio recording of consultations. Formal trials of this type of approach have shown promise in the oncology setting, with respect to improving patients' information needs, their satisfaction with patient-professional communication and recall of information. ${ }^{40-42}$ Whether these findings can be extrapolated to specific apps is unclear.

Many of the apps we reviewed attempted to influence medical care by suggesting that self-monitoring reports be shared with clinicians. Patient-reported outcome monitoring has been shown to improve patient satisfaction with care in the oncology setting, and to increase the number of patient outcomes that are discussed during consultations. ${ }^{26}{ }^{27}$ However, any effects are likely to be contingent on how the data are used during clinical encounters and what data are collected. ${ }^{43}$ Scientific trials tend to use validated questionnaires, as opposed to the, often generic, tools present within apps.

There seems to be a widely held assumption in symptom management apps that providing patients with simple graphical summaries of their self-reported symptom data will afford insights that could improve symptom management. Conversely, there is a danger that apps could increase the work and burden of cancer
Box 1 Four D's to discuss with patients if they are considering using a health app

Does something useful—does it solve a problem you are having? Design-are there screenshots that summarise the content and give you an impression of how you would use the app?

Developer-do you recognise a credible organisation/source behind the app, and do links to the developer website work?

Data-does the app ask you for personal information that you would prefer not to be shared with others or provide a transparent description of how data will be used and shared?

survivorship activities without resultant benefits to the user. We noted close parallels between categories of app content (table 3), and models of treatment burden in other chronic conditions. ${ }^{29-31}$

The app market is a potentially challenging environment for patients and clinicians to navigate in terms of judging app quality, effectiveness, clinical utility and data privacy. It may be that app stores themselves should be asked to take more responsibility for the content of the apps they offer. Several high-profile scandals, for example, Cambridge Analytica allegedly using Facebook data to influence election results, ${ }^{44}$ and suicides potentially linked to social media use ${ }^{45}$ have led to increased public scrutiny surrounding the social responsibilities of technology providers. With respect to app stores, existing legislation, such as trading standards regulations that prevent false or misleading advertising, and General Data Protection Regulation might be enforced to protect consumers. The National Health Service has also started a library of approved apps that have been screened against quality criteria. ${ }^{46}$ Three of the apps reviewed here, BECCA, OWISE breast cancer and Untire: Beating cancer fatigue, appear in the library.

While app stores continue to offer low-quality and potentially exploitative apps, we propose a rudimentary checklist (box 1), the 'Four D's', which might be used by patients before downloading a health app. The checklist was derived pragmatically, based on our experiences of conducting this review, and on the existing literature/ guidelines discussed above..$^{33} 35389$

Intuitively, we considered a fifth 'D'-Downloads, in which the number of downloads and positive/detailed consumer reviews might serve as an indicator of quality and trustworthiness. Box 1 Apps by reputable organisations tended to be highly downloaded, but we also found highly downloaded apps which seemed to be of low quality for example, Cancer Curing Foods, offering 'top 10 fruits, vegetables and foods that can cure cancer' had been downloaded over 10000 times. We also considered that some app reviews could be false or purposefully misleading. The association between number of downloads and objective measures of quality deserve further attention. 


\section{Strengths and limitations}

The app market is changing rapidly-more apps are being added to app stores every day, and it is also possible for developers to delete apps from on-line stores. We have presented a snapshot of what was available between September 2018 and May 2019. The main limitation of this review is that we did not download and interact with individual apps. To have done so would have added considerable time to the review process (which was time-sensitive, given the changing nature of the market), and would have also involved buying several apps that looked to have limited content (eg, 'Don't die 2' retailed at $£ 299.99)$. Our content analysis is based on what was stated in on-line descriptions, and may underestimate content contained within the apps. We did not register a review protocol, which is a relatively new requirement in updated Preferred Reporting Items for Systematic Reviews and Meta-Analyses guidelines for scoping reviews, which were published after we started this review. ${ }^{47}$

App stores are commercial entities and are not searchable in the same way as databases of published medical literature. We fully expect that other relevant apps exist which have not been identified by our searches. Furthermore, it is not possible to save or export searches. Apps are displayed in an order that is determined by on-line stores, and, to the best of our knowledge, the exact sort algorithms used by stores are not in the public domain. This makes searches difficult to accurately reproduce, and made it difficult to involve two authors in all stages of the app selection process. Nevertheless, where possible, we have adopted principles of systematic reviewing. We are confident that we have identified apps in a systematic and unbiased way and have characterised a large spectrum of currently available apps.

\section{CONCLUSIONS}

Apps exist that cover a large spectrum of cancer survivorship activities: key components are information provision, storing personal summaries and self-monitoring. The effects of such apps on clinical consultations, patient work/burden and clinical outcomes merit further attention. Most apps are developed by commercial organisations and promises of empowerment in the 'fight' against cancer are tempered by the potential for exaggerated claims and exploitation.

Contributors RA conceptualised this review, designed the review, undertook searches, assessed the apps for inclusion/exclusion, undertook data extraction and wrote the paper. DM undertook scoping searches, helped refine the search criteria, checked the final apps fulfilled inclusion/exclusion criteria and performed independent dual data extraction and content coding. He contributed to drafts of the paper and revised the article critically. DP assisted with thematic analysis and revised the article critically. PM contributed to drafts of the paper and revised the article critically.

Funding This work was supported by NHS Grampian Pump Priming funding, grant reference RG14437-11. DP is supported by the strategic research programme funded by the Scottish Government's Rural and Environment Science and Analytical Services Division.

Competing interests None declared.
Patient consent for publication Not required.

Provenance and peer review Not commissioned; externally peer reviewed.

Data availability statement All data relevant to the study are included in the article or uploaded as online supplementary information.

Open access This is an open access article distributed in accordance with the Creative Commons Attribution Non Commercial (CC BY-NC 4.0) license, which permits others to distribute, remix, adapt, build upon this work non-commercially, and license their derivative works on different terms, provided the original work is properly cited, appropriate credit is given, any changes made indicated, and the use is non-commercial. See: http://creativecommons.org/licenses/by-nc/4.0/.

\section{ORCID iDs}

Rosalind Adam http://orcid.org/0000-0003-3082-6578

Peter Murchie http://orcid.org/0000-0001-9968-5991

\section{REFERENCES}

1. National Cancer Institute. Cancer statistics [Internet]. Available: https://www.cancer.gov/about-cancer/understanding/statistics [Accessed 1 Feb 2019].

2. Maddams J, Utley M, Møller H. Projections of cancer prevalence in the United Kingdom, 2010-2040. Br J Cancer 2012;107:1195-202.

3. Adam R, Watson E. The role of primary care in supporting patients living with and beyond cancer. Curr Opin Support Palliat Care 2018;12:261-7.

4. Glare PA, Davies PS, Finlay E, et al. Pain in cancer survivors. J Clin Oncol 2014;32:1739-47.

5. Hoekstra RA, Heins MJ, Korevaar JC. Health care needs of cancer survivors in general practice: a systematic review. BMC Fam Pract 2014;15:94.

6. Elliott J, Fallows A, Staetsky L, et al. The health and well-being of cancer survivors in the UK: findings from a population-based survey. Br J Cancer 2011;105(Suppl 1):S11-20.

7. Nekhlyudov L, O'malley DM, Hudson SV. Integrating primary care providers in the care of cancer survivors: gaps in evidence and future opportunities. Lancet Oncol 2017;18:e30-8.

8. Escriva Boulley $\mathrm{G}$, Leroy $\mathrm{T}$, Bernetière $\mathrm{C}$, et al. Digital health interventions to help living with cancer: a systematic review of participants' engagement and psychosocial effects. Psychooncology 2018;27:2677-86.

9. Groen WG, Kuijpers W, Oldenburg HS, et al. Empowerment of cancer survivors through information technology: an integrative review. $J$ Med Internet Res 2015;17:e270.

10. Business of Apps. App download and usage statistics (2018) [Internet], 2018. Available: http://www.businessofapps.com/data/ app-statistics/ [Accessed 14 Feb 2019].

11. Burki TK. Cancer apps. Lancet Oncol 2013;14:580-1.

12. IQVIA Institute for Human Data Science. The growing value of digital health [Internet]. Available: https://www.iqvia.com/institute/reports/ the-growing-value-of-digital-health [Accessed 19 Aug 2019].

13. Liquid State. The rise of mHealth apps: a market snapshot [Internet], 2018. Available: https://liquid-state.com/mhealth-apps-marketsnapshot/ [Accessed 29 May 2019].

14. Cox A, Lucas G, Marcu A, et al. Cancer survivors' experience with telehealth: a systematic review and thematic synthesis. J Med Internet Res 2017;19:e11.

15. Dickinson R, Hall S, Sinclair JE, et al. Using technology to deliver cancer follow-up: a systematic review. BMC Cancer 2014;14:311.

16. Abroms LC, Lee Westmaas J, Bontemps-Jones J, et al. A content analysis of popular smartphone apps for smoking cessation. Am J Prev Med 2013;45:732-6.

17. Schoffman DE, Turner-McGrievy G, Jones SJ, et al. Mobile apps for pediatric obesity prevention and treatment, healthy eating, and physical activity promotion: just fun and games? Trans/ Behav Med 2013;3:320-5.

18. Azar KMJ, Lesser LI, Laing BY, et al. Mobile applications for weight management: theory-based content analysis. Am J Prev Med 2013;45:583-9.

19. Bender JL, Yue RYK, To MJ, et al. A lot of action, but not in the right direction: systematic review and content analysis of smartphone applications for the prevention, detection, and management of cancer. J Med Internet Res 2013;15:e287.

20. Kassianos AP, Emery JD, Murchie P, et al. Smartphone applications for melanoma detection by community, patient and generalist clinician users: a review. Br J Dermatol 2015;172:1507-18.

21. Vollmer Dahlke D, Fair K, Hong YA, et al. Apps seeking theories: results of a study on the use of health behavior change theories in cancer survivorship mobile apps. JMIR Mhealth Uhealth 2015;3:e31. 
22. Abraham C, Michie S. A taxonomy of behavior change techniques used in interventions. Health Psychol 2008;27:379-87.

23. Levac D, Colquhoun H, O'Brien KK. Scoping studies: advancing the methodology. Implementation Sci 2010;5.

24. Hsieh H-F, Shannon SE. Three approaches to qualitative content analysis. Qual Health Res 2005;15:1277-88.

25. Arksey H, O'Malley L. Scoping studies: towards a methodological framework. Int J Soc Res Methodol 2005;8:19-32.

26. Davis K, Drey N, Gould D. What are scoping studies? A review of the nursing literature. Int J Nurs Stud 2009;46:1386-400.

27. Joffe H, Yardley L. Research methods for clinical and health psychology [Internet]. SAGE, 2004: 1-248. https://books.google. $\mathrm{com} /$ books?hl=en\&lr=\&id=SHiUvmKzuFwC\&pgis $=1$

28. The Psychologist. Methods: teaching thematic analysis [Internet] Available: http://thepsychologist.bps.org.uk/volume-26/edition-2/ methods-teaching-thematic-analysis [Accessed 8 Feb 2017].

29. Zick SM, Sen A, Wyatt GK, et al. Investigation of 2 types of selfadministered Acupressure for persistent cancer-related fatigue in breast cancer survivors: a randomized clinical trial. JAMA Oncol 2016;2:1470-6.

30. UKONS. UK oncology nurses society triage tool [Internet]. Available: http://necn.nhs.uk/wp-content/uploads/2018/05/UKONS-Tool-.pdf [Accessed 6 Jun 2019].

31. Cancer Research UK. May I take your metaphor? - how we talk about cancer [Internet]. Available: https://scienceblog. cancerresearchuk.org/2015/09/28/may-i-take-your-metaphor-howwe-talk-about-cancer/ [Accessed 6 Jun 2019].

32. American Society of Clinical Oncology. Guidelines on survivorship care [Internet], 2014. Available: https://www.asco.org/practiceguidelines/cancer-care-initiatives/prevention-survivorship/ survivorship-compendium-0 [Accessed 23 Apr 2018].

33. Royal College of Physicians. Using apps in clinical practice guidance [Internet], 2015. Available: https://www.rcplondon.ac.uk/guidelinespolicy/using-apps-clinical-practice-guidance [Accessed $18 \mathrm{Apr}$ 2019].

34. Medicines \& Healthcare products Regulatory Agency. Guidance: medical device stand-alone software including apps (including IVDMDs) [Internet], 2018. Available: https://assets.publishing.service. gov.uk/government/uploads/system/uploads/attachment_data/file/ 717865/Software_flow_chart_Ed_1-05.pdf

35. US Departments of Health and Human Services Food and Drug Adminsitration, Center for Devices and Radiological Health C for BE and R. Mobile medical applications. Guidelines for Industry and Food and Drug Adminsitration staff [Internet], 2015. Available: https://www. fda.gov/downloads/MedicalDevices/DeviceRegulationandGuidance/ GuidanceDocuments/UCM263366.pdf
36. van Velthoven $\mathrm{MH}$, Wyatt JC, Meinert E, et al. How standards and user involvement can improve APP quality: a lifecycle approach. Int $J$ Med Inform 2018;118:54-7.

37. National Information Board UG. Personalised health and care 2020 Using data and technology to transform outcomes for patients and citizens [Internet], 2014. Available: https://assets.publishing.service. gov.uk/government/uploads/system/uploads/attachment_data/file/ 384650/NIB_Report.pdf

38. Wyatt JC. How can clinicians, specialty societies and others evaluate and improve the quality of apps for patient use? BMC Med 2018; $16: 225$

39. Grundy Q, Chiu K, Held F, et al. Data sharing practices of medicines related apps and the mobile ecosystem: traffic, content, and network analysis. BMJ 2019;364:1920.

40. Wolderslund M, Kofoed P-E, Holst R, et al. Digital audio recordings improve the outcomes of patient consultations: a randomised cluster trial. Patient Educ Couns 2017:100:242-9.

41. Clayton JM, Butow PN, Tattersall MHN, et al. Randomized controlled trial of a prompt list to help advanced cancer patients and their caregivers to ask questions about prognosis and end-of-life care. $J$ Clin Oncol 2007;25:715-23.

42. Amundsen A, Bergvik S, Butow P, et al. Supporting doctorpatient communication: providing a question prompt list and audio recording of the consultation as communication AIDS to outpatients in a cancer clinic. Patient Educ Couns 2018;101:1594-600.

43. Adam R, Burton CD, Bond CM, et al. Can patient-reported measurements of pain be used to improve cancer pain management? A systematic review and meta-analysis. BMJ Support Palliat Care 2017;7

44. BBC News. Cambridge Analytica: the story so far [Internet]. Available: https://www.bbc.co.uk/news/technology-43465968 [Accessed 19 Aug 2019].

45. The Guardian. Social media urged to take 'moment to reflect' after girl's death | Media [Internet]. Available: https://www.theguardian. com/media/2019/jan/30/social-media-urged-to-take-moment-toreflect-after-girls-death [Accessed 19 Aug 2019].

46. NHS Digital. How we assess health apps and digital tools [Internet]. Available: https://digital.nhs.uk/services/nhs-apps-library/guidancefor-health-app-developers-commissioners-and-assessors/how-weassess-health-apps-and-digital-tools\#how-the-assessment-works [Accessed 19 Aug 2019].

47. Tricco AC, Lillie E, Zarin W, et al. PRISMA extension for scoping reviews (PRISMA-ScR): checklist and explanation. Ann Intern Med 2018;169:467. 